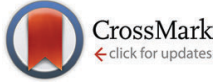

Cite this: J. Mater. Chem. B, 2015, 3, 7745

Received 24th June 2015,

Accepted 1st September 2015

DOI: $10.1039 / c 5 t b 01232 e$

www.rsc.org/MaterialsB

\section{Determination of the shell growth direction during the formation of silica microcapsules by confocal fluorescence microscopy}

\author{
Judith van Wijk, $\dagger^{a}$ Joris W. O. Salari, $\stackrel{\ddagger}{a}^{a}$ Jan Meuldijk and Bert Klumperman* ${ }^{\mathrm{b}}$
}

\begin{abstract}
A novel procedure was developed to determine the direction of silica growth during the formation of a silica shell around aqueous microdroplets in water-in-oil Pickering emulsions. Two fluorescently labeled silica precursors were added consecutively and the resulting microcapsules were visualized via confocal fluorescence microscopy, allowing the conclusion that the locus of reaction moves in the positive radial direction, i.e. from the inside to the outside. A consequence of the growth direction is that water has to diffuse through the shell to participate in the reaction on the outer surface of the shell.
\end{abstract}

\section{Introduction}

For proper microencapsulation there is a demand for precise control of synthetic procedures in order to create capsules with well-defined architectures. This aim is important in view of the various potential applications, which can be roughly divided into protection and controlled release applications. ${ }^{1-5}$ A further goal is to avoid contamination of the core in order to keep the encapsulated active ingredients unaffected. To accomplish these two imperative demands it is necessary to fully understand the process of capsule formation. Protection applications are, for example, the microencapsulation of phase change materials and probiotic bacteria. ${ }^{2,6-10}$ Controlled release applications are for instance the controlled release of drugs inside the body and the controlled release of a selective reactant in a reaction mixture. ${ }^{4,5,11,12}$ Various applications ask for different types of capsules. In the case of controlled release applications the capsules should have a controllable permeability and for protection application the microcapsule should seal the active ingredient from the environment. Besides the capsule properties, also the encapsulation procedure is important. For example, bacteria are sensitive towards chemicals, $\mathrm{pH}$ and temperature changes in their surroundings. Silica as capsule material is suitable for a number of applications, since it is chemically and biologically inert, nontoxic and mechanically and thermally stable. ${ }^{13-15}$ Tetraethyl orthosilicate (TEOS) is a well known

\footnotetext{
${ }^{a}$ Eindhoven University of Technology, Department of Chemical Engineering and Chemistry, P.O. Box 513, 5600 MB Eindhoven, The Netherlands

${ }^{b}$ Stellenbosch University, Dept. Chemistry and Polymer Science, Private Bag X1, Matieland 7602, South Africa. E-mail: bklump@sun.ac.za

$\dagger$ Current address: Eastman Chemical Company, Herculesweg 35, 4338 PL, Middelburg, The Netherlands.

\$ Current address: TNO, de Rondom 1, 5612 AP, Eindhoven, The Netherlands.
}

silica precursor which reacts by polycondensation with water to silica. The reaction is catalyzed by a base or an acid. Typically, capsules formation via polycondensation reactions occurs by placing the necessary reactants in opposite phases. Therefore, the reactants only meet at the interface. So the interface is where the reaction takes place and a capsule is produced. ${ }^{13,16-19}$ But, a more profound understanding of the shell formation, instead of just placing the reactants in opposite phases, would lead to a better design of the encapsulation procedure. This is especially valuable in case $e . g$. the core should not be contaminated or if the shell should have a certain thickness. Various hypotheses have been put forward regarding the mechanism of formation of such microcapsules. ${ }^{13,20,21}$ When producing silica microcapsules from TEOS, most hypotheses are based on the notion that the hydrophilicity of the (oil-soluble) silica precursor changes as a result of the hydrolysis reaction during the course of the interfacial reaction. As a consequence, the silica precursor can either react at the interface to form a microcapsule or dissolve in the aqueous phase. However, experimental proof regarding the precise mechanism of shell formation is lacking.

In this work, silica microcapsules were produced by templating Pickering emulsion droplets in a water-in-oil dispersion. In view of the mechanism of shell formation, we present a novel method to determine the direction of silica growth, using confocal microscopy imaging. A fluorescein-labeled and a rhodamine-labeled silica precursor are added consecutively during the capsule synthesis. The direction of growth of the shell is determined by observation of the relative location of the dyes in the shell, using confocal fluorescence microscopy. Knowledge of the growth direction leads to a solid conclusion about the locus of reaction and provides insight into transport of reactants and products through the shell during the silica microcapsule formation. 


\section{Results and discussion}

Silica microcapsules, with an aqueous core, were synthesized by polymerization of tetraethoxy orthosilicate (TEOS) in the presence of inverse Pickering emulsion droplets as a template and by using $n$-hexylamine as an amphiphilic catalyst. ${ }^{22}$ During this encapsulation method, silica particles are initially ultrasonically dispersed in a mixture of toluene and $n$-hexylamine. Water is emulsified in the silica particle dispersion by the use of a rotor-stator (IKA Ultra Turrax T18 basic) high-shear mixing device to yield a water-in-oil emulsion. Such a so-called inverse Pickering emulsion is most stable when the particles have a three-phase contact angle between $94^{\circ}$ and $110^{\circ} \cdot{ }^{23-26}$ Silica microparticles are known to be very hydrophilic, with a contact angle $<10^{\circ}$ and are therefore considered not to be suitable to stabilize an inverse Pickering emulsion. Generally, if silica microparticles are to be used in an inverse Pickering emulsion

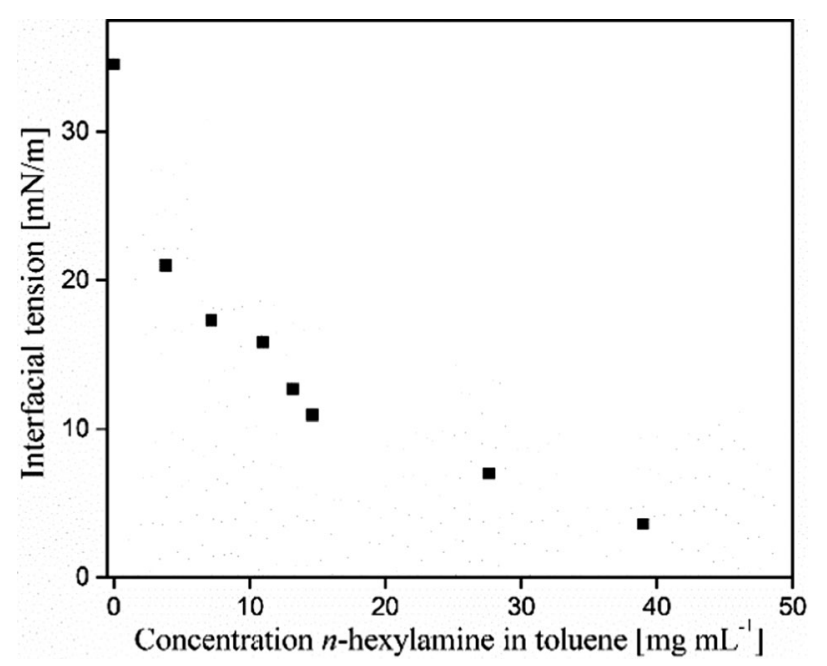

Fig. 1 Interfacial tension between water and toluene as a function of the $n$-hexylamine concentration in toluene. they first need to be hydrophobized. ${ }^{27,28}$ However, $n$-hexylamine has amphiphilic properties, see Fig. 1. When an amphiphile is added to a Pickering emulsion, the stability will be determined by synergistic interactions of the amphiphilic molecules with the

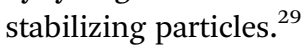

As a result of the synergistic interactions between the silica particles and $n$-hexylamine, it proved to be possible to use hydrophilic silica particles to stabilize a Pickering emulsion (Fig. 2A and B) as well as to stabilize an inverse Pickering emulsion (Fig. 2C-E). This phenomenon is also named phase inversion as a result of the $n$-hexylamine concentration. ${ }^{30}$ Fig. 2 shows light microscopy images of five different Pickering emulsions produced. The volume ratio of water to toluene was always $1: 1 \mathrm{v} / \mathrm{v}$, the $n$-hexylamine concentration increased from 5 to $57 \mathrm{mg} \mathrm{mL}^{-1}$. Consequently, the emulsions were stabilized by the same number of silica particles, but every time with a different $n$-hexylamine concentration in the toluene phase.

An inverse Pickering emulsion was loaded in a reactor with overhead stirring and TEOS was added subsequently in order to grow a silica shell. Since water, as a reactant, is present in the emulsion droplet and TEOS is in the continuous organic phase, the precursor and water have the highest probability to react at the interface, see Fig. 3. However, it has been shown in previous work that the precursor can diffuse into the water droplet upon hydrolysis, and the reaction no longer exclusively takes place at the interface. ${ }^{22}$ On the other hand, it has also been shown that the use of $n$-hexylamine strictly directs the reaction to the interface and not to the inside of the emulsion droplets. ${ }^{22}$

The silica particles that were used for the inverse Pickering emulsions were synthesized using a seeded Stöber reaction. ${ }^{31,32}$ This approach resulted in silica microparticles with a narrow size distribution $D_{\mathrm{Z}} / D_{\mathrm{V}} \approx 1.01$, (Fig. $4 \mathrm{~A}$ ). The particle concentration in the Pickering emulsion to produce a certain droplet size (assuming close-packing on the interface), and the concentration of TEOS to produce a certain shell thickness were calculated as previously reported. $^{22}$ To produce a Pickering emulsion with a narrow

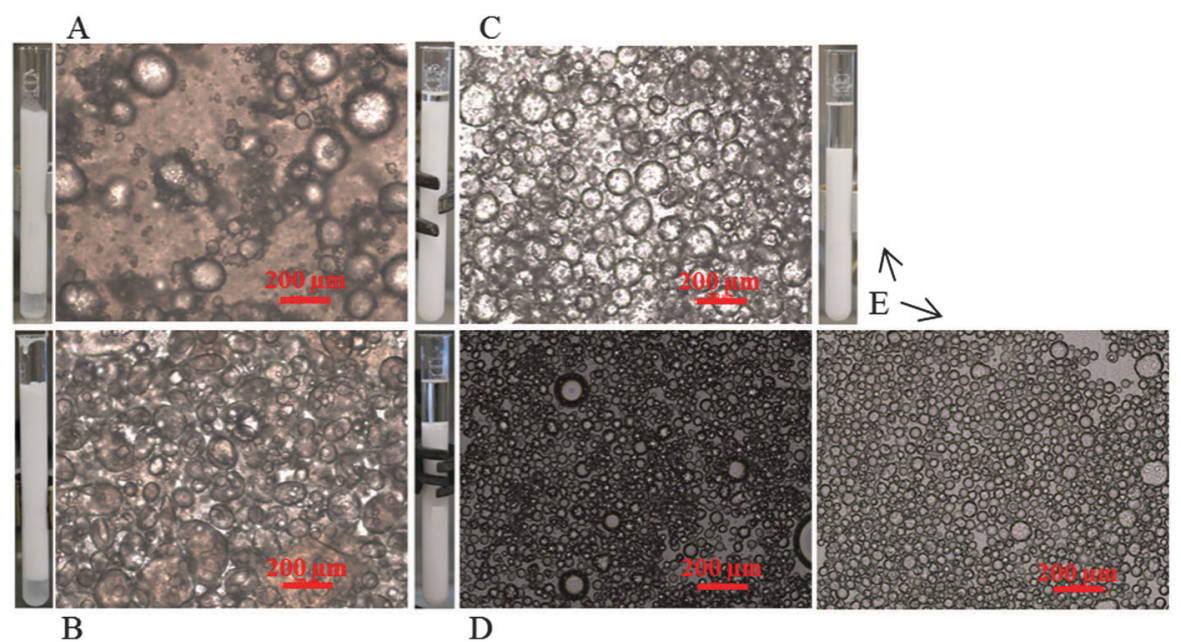

Fig. 2 Phase inversion as a result of $n$-hexylamine concentration. Light microscopy images, of Pickering emulsions (A and B) and inverse Pickering emulsions (C to E) stabilized with silica particles. The concentrations of $n$-hexylamine in the toluene phase were $5,15,31,51$ and $57 \mathrm{mg} \mathrm{mL}^{-1}$, respectively. The other concentrations were kept the same for all emulsions. The volume ratio of water to toluene is $1: 1 \% \mathrm{v} / \mathrm{v}$. 


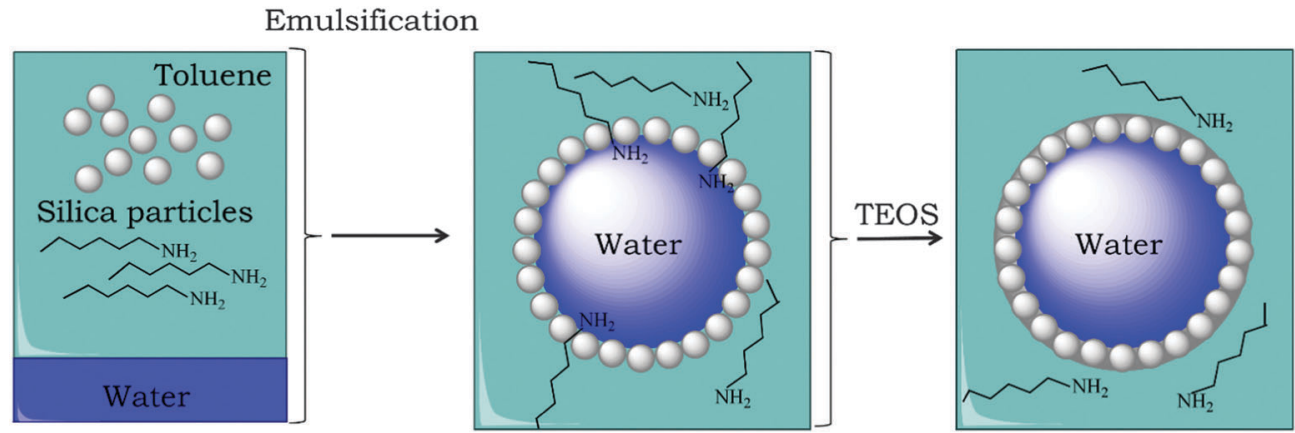

Fig. 3 Schematic overview of the synthesis of silica microcapsules. (left) all components of the Pickering emulsion are added together. (middle) Application of shear force results in the formation of silica-particle-stabilized water droplets. (right) Capsule formation occurs after the addition of TEOS.

droplet size distribution, the particles should be well dispersed in the oil phase before emulsification. So it is possible to form a close packing at the oil-water interface. This resulted in an emulsion with a droplet size distribution characterized by $D_{\mathrm{z}} / D_{\mathrm{v}} \approx 1.1$. After the addition of TEOS, a solid amorphous silica shell was formed around the emulsion droplets. The size distribution of the capsules was identical to that of the original Pickering emulsion droplet templates (Fig. 4B and C). The SEM images show that the majority of the silica microcapsules are intact, even under the high vacuum conditions required for SEM, which means they are robust. The capsules that are broken have remarkably spherical fractures. We speculate that the formation of these spherical fractures is caused by a pressure difference that is generated upon the evaporation of water outwards and slow diffusion of air inwards. This causes a pressure difference resulting in the spherical fractures.

During the shell formation it was anticipated that at a certain moment, the shell would form a barrier between the two phases and would cause the reaction to stop or at least to slow down significantly. Instead, the shell has a thickness of a few micrometers within a reaction time of $6 \mathrm{~h}$ and the thickness is consistent with the amount of TEOS that was added. This result indicates that the reaction did not stop but the shell continued to grow until all TEOS was depleted.

Part of the observations described above can be explained if the shell is growing from the inside to the outside. In that situation, water has to diffuse through the already formed shell to participate in the reaction. This means that the time constant for diffusion of water to the locus of reaction increases upon increasing shell thickness. As a consequence, the shell growth rate decreases with time, and beyond a certain thickness shell growth will apparently stop.

Confocal Fluorescence Microscopy was used to unequivocally determine the growth direction of the shell. A fluorescein-labeled and a rhodamine-labeled triethoxysilicate precursor were added consecutively during the capsule synthesis and the direction of growth was determined by observation of the relative location of the dyes in the shell, using confocal fluorescence microscopy. Through the synthesis of the labeled microcapsules, TEOS was added in 5 aliquots and to the second and fourth aliquot, one of
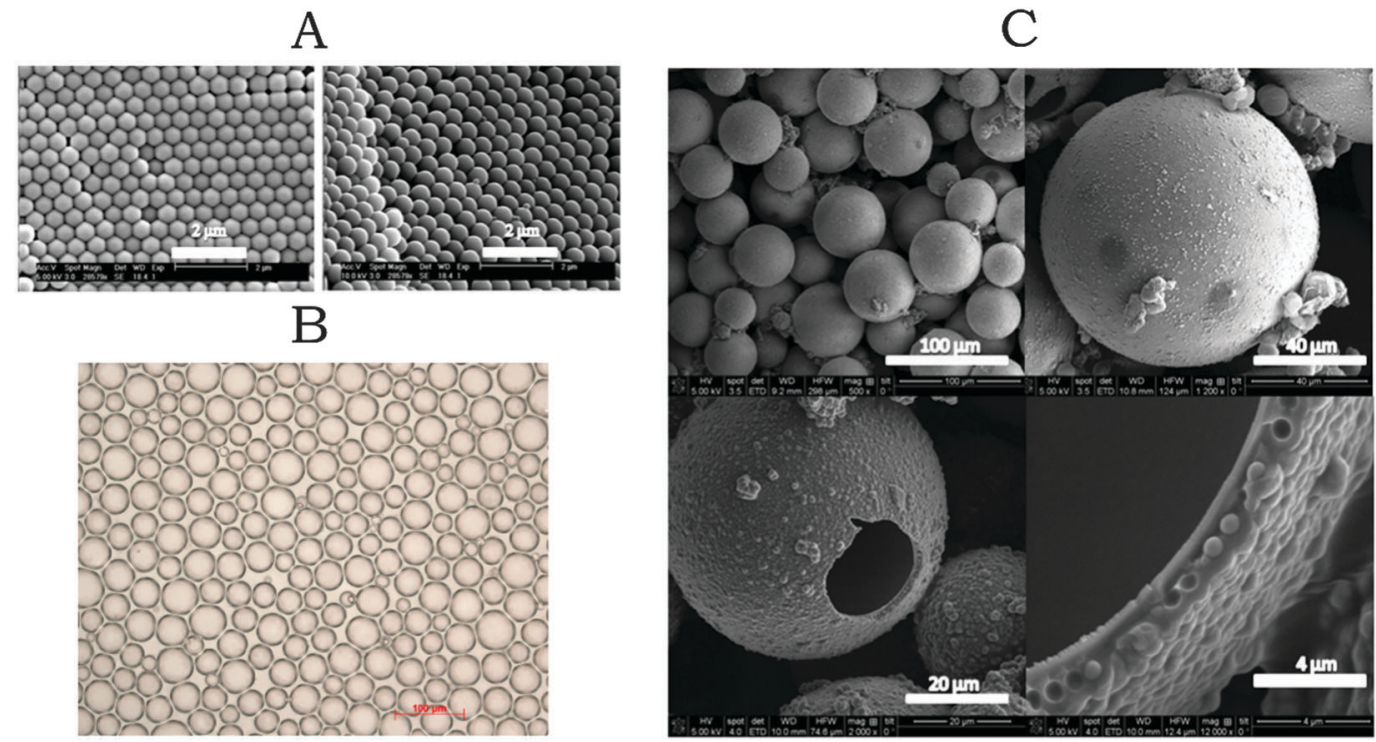

Fig. 4 Images of various stages during the silica microcapsule synthesis. SEM images of primary silica microparticles (A), light microscopy image of the Pickering emulsion droplets (B) and SEM images of silica microcapsules (C). 


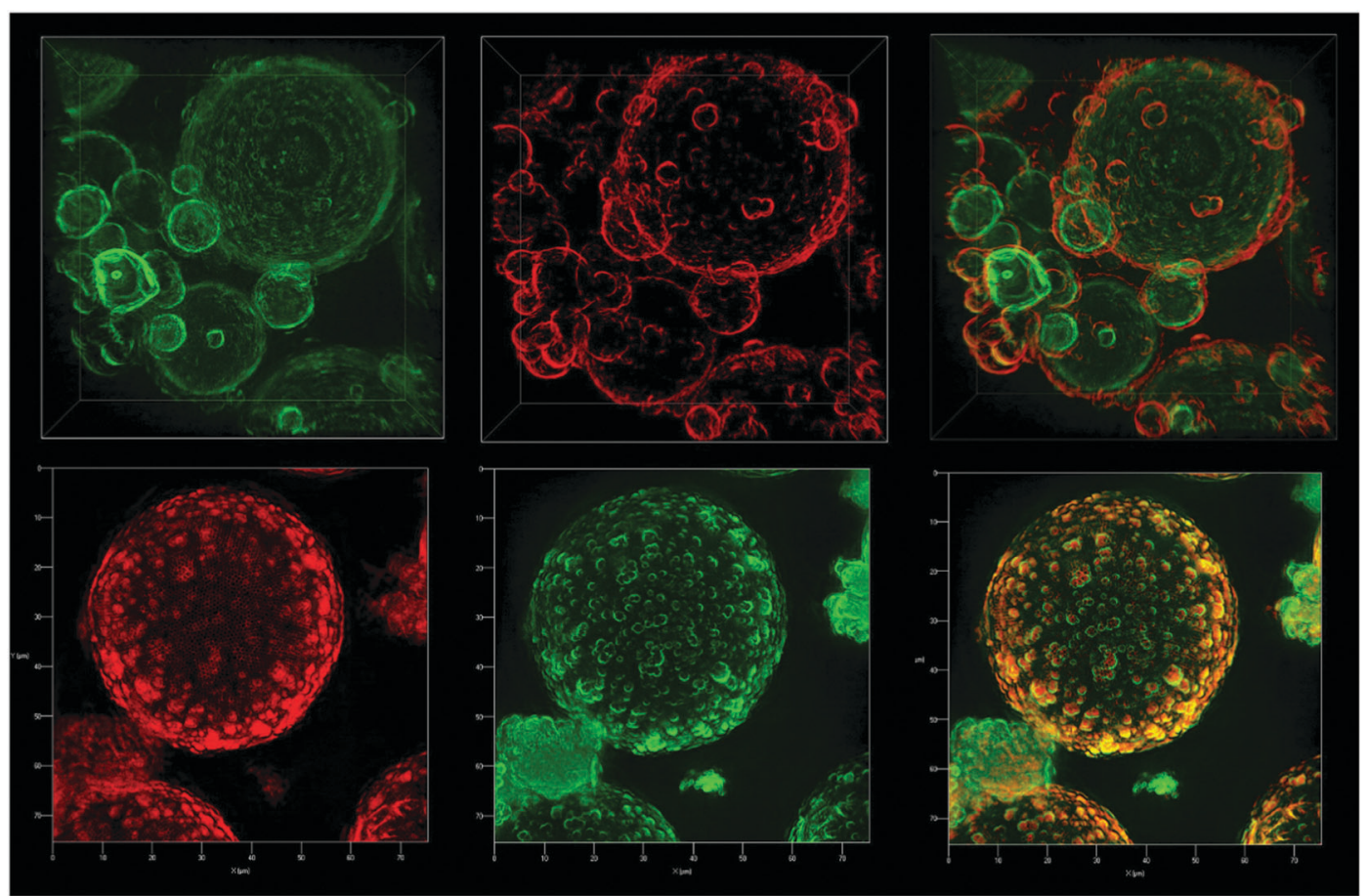

Fig. 5 Fluorescence confocal microscopy images of rhodamine-fluorescein-labeled silica microcapsules (top images) and fluorescein-rhodaminelabeled silica microcapsules (bottom images). The two dye-labeled triethoxy silicate precursors have been added in aliquots two and four out of five total aliquots of TEOS addition.

the silica precursor dyes was added. Every new addition was done after at least 3 hours of reaction time, to ensure sufficient conversion of the previously added TEOS. The labeled silica precursors were synthesized according to a literature procedure by the addition reaction between fluorescein isothiocyanate or rhodamine B isothiocyanate and aminopropyltriethoxysilane in ethanol. ${ }^{33,34}$ The amount of labeled silica precursors added was in the order of milligrams, relative to the total newly formed silica, which was in the order of grams. Both dyes were dissolved in ethanol upon addition, because they were taken directly from the crude reaction mixture. The extra ethanol that was added with the dyes did not influence the interfacial reaction, since ethanol is produced in much larger quantities during the course of the interfacial reaction. Also, when not reacted with aminopropyltriethoxysilane, the dyes do not chemically or physically adsorb to the newly formed silica shell. ${ }^{33,34}$ Before the rhodaminefluorescein-labeled silica microcapsules were imaged by confocal fluorescence microscopy, they were washed with ethanol twice to get rid of un-reacted dyes. As a result, when the fluorescein-labeled silica precursor was added before the rhodamine-labeled precursor, the confocal fluorescence microscope images clearly show that the inside of the silica microcapsules is green fluorescein-labeled and the outside is red rhodamine-labeled and vice versa, (Fig. 5). This provides unambiguous proof that the shell grows from the inside to the outside. Besides that, the silica microparticles that initially stabilized the Pickering emulsion can be distinguished from the newly formed fluorescein-labeled silica shell. This is in agreement with the SEM images, Fig. 4. At a later stage, the newly formed silica engulfs the particles and silica formation takes place on the whole surface of the shell. This can be observed via the dye that was added last and that as a result covers the entire surface. Also visible, is the difference in shell morphology between the inner and outer side of the microcapsules, the outer side appears to have a more irregular morphology.

A direct consequence of the growth direction is that the reaction takes place on the exterior surface of the microcapsules. Hence, water has to diffuse through the growing shell to participate in the reaction. Initially, all necessary components for the reaction are in contact with each other at the interface and a very thin shell is produced. After that, water diffuses through the shell and as a result the shell can grow thicker, from the inside to the outside. Because the reaction is taking place on the outside of the capsules, there is a minimum of contamination of the dispersed phase.

\section{Conclusion}

The direction of growth of a silica shell around an inverse Pickering emulsion droplet has been investigated with the aid of fluorescently labeled silica precursors. Confocal fluorescence microscopy on the resulting fluorescently labeled microcapsules allowed us to determine the relative position of the dyes in the shell. Based on these results it was unambiguously shown that the silica shell grows from the inside to the outside, and silica formation takes place on the exterior surface of the shell. This procedure is possibly useful for other studies on growth direction and morphology in the micrometer and nanometer size-range. 


\section{Experimental}

\section{Materials}

All chemicals were used as received, unless indicated otherwise. Tetraethyl orthosilicate (TEOS), (3-aminopropyl)triethoxysilane (99\%) and rhodamine $\mathrm{B}$ isothiocyanate $\left(\mathrm{C}_{29} \mathrm{H}_{30} \mathrm{ClN}_{3} \mathrm{O}_{3} \mathrm{~S}, \lambda_{\text {ex }}\right.$ $543 \mathrm{~nm}, \lambda_{\mathrm{em}} 580 \mathrm{~nm}$ in methanol) were purchased from Sigma Aldrich. $n$-Hexylamine and ethanol anhydrous (99.8\%) purchased from Merck-Chemicals. Toluene (AR) was purchased from Biosolve. Fluorescein 5(6)-isothiocyanate $\left(\mathrm{C}_{21} \mathrm{H}_{11} \mathrm{NO}_{5} \mathrm{~S}, \lambda_{\mathrm{ex}}=\right.$ $495 \mathrm{~nm}, \lambda_{\mathrm{em}}=525 \mathrm{~nm}$ ) (90\% HPLC) was purchased from Fluka. The water used was double de-ionized water produced by an Elix Millipore purification system.

\section{Monodisperse silica microparticles}

The monodisperse silica microparticles were prepared by a seeded Stöber polymerization technique. ${ }^{32}$ First the seed particles were synthesized via the Stöber method. ${ }^{31}$ In short, TEOS (7 g, $33.6 \mu \mathrm{mol})$ and water $(5 \mathrm{~mL}, 278 \mu \mathrm{mol})$ were solubilized in $100 \mathrm{~mL}$ ethanol, in a three-neck round-bottom flask of $250 \mathrm{~mL}$ at $25{ }^{\circ} \mathrm{C}$ stirred by a magnetic stirrer. After $10 \mathrm{~min}$, ammonia $(15 \mathrm{~mL}, 25 \%)$ was added to base-catalyze the reaction. After at least 6 hours of reaction time tetraethyl orthosilicate (TEOS) ( $2 \mathrm{~g}, 9.6 \mu \mathrm{mol}$ per addition) was added multiple times. Every new $2 \mathrm{~g}$ TEOS addition was done at least 6 hours after the previous addition, to ensure full conversion of the previously added TEOS. The total amount of TEOS was adjusted so the monodisperse particles would have a diameter of $500 \mathrm{~nm}$ (if $\rho=2.15 \mathrm{~g} \mathrm{~cm}^{-3}$ ). Once the reaction was completed, the particles were left to settle, after which the liquid phase was decanted and the particles were air-dried. According to Bogush et al. ${ }^{32}$ the size of the particles can be related to the total number of moles of TEOS added: $d=d_{0}\left(N_{\text {TEOS }} / N_{\text {TEOS }, 0}\right)^{1 / 3}$, in which $d$ is the particle diameter, $d_{0}$ is the average seed particle diameter, $N_{\text {TEOS }, 0}$ is the number of moles TEOS added to produce the seed particles and $N_{\text {TEOS }}$ is the total number of moles TEOS added to the dispersion including. $N_{\text {TEOS, } 0}$.

\section{Pickering emulsion}

The inverse Pickering emulsion was prepared by first redispersing the prepared and dried silica microparticles in a solution of toluene and $n$-hexylamine, using ultrasonication, until a translucent dispersion was produced. The amounts of toluene, the silica microparticles and water were calculated according to Salari et al. ${ }^{35}$ Typically, about $16 \mathrm{~mL}$ of water, $2.5 \mathrm{~g}$ of silica particles (diameter is $500 \mathrm{~nm}$ ) and $60 \mathrm{~mL}$ of toluene was used. For all prepared Pickering emulsions, formulations were chosen so that the average droplet diameter would be $50 \mu \mathrm{m}$. A number of concentrations of $n$-hexylamine in toluene was used. $n$-Hexyalmine in toluene concentration of 5 and $57 \mathrm{mg} \mathrm{mL} \mathrm{mL}^{-1}$ resulted in a stable inverse Pickering emulsion (Fig. 2). The inverse Pickering emulsion was produced by applying high shear via a rotor stator device (Ultra Turrax ${ }^{\circledR}$ T18 Basic) operating at $20000 \mathrm{rpm}$. The time needed to obtain a proper emulsion was $2 \mathrm{~min}$.

\section{Synthesis of silica microcapsules}

The silica microcapsules were produced by the addition of TEOS to the inverse Pickering emulsion. The reaction proceeded under continuous overhead stirring at $25{ }^{\circ} \mathrm{C}$. The amount of TEOS was adjusted to produce microcapsules with a certain shell thickness, calculated according to literature. ${ }^{22}$

\section{The rhodamine-fluorescein-labeled silica}

Microcapsules shown in Fig. 5 were produced with an emulsion consisting of silica microparticles (2 g, diameter $830 \mathrm{~nm}$ ), water $(16 \mathrm{~mL})$ and toluene with $52 \mathrm{mg} \mathrm{mL}{ }^{-1} n$-hexylamine $\left(0.51 \mu \mathrm{mol} \mathrm{mL}{ }^{-1}\right)(150 \mathrm{~mL})$. After the Pickering emulsion was produced, TEOS was added in 5 batches of 4, 4, 3.7, 4.1 and $2 \mathrm{~g}$ $(19,19,18,20$ and $9.6 \mu \mathrm{mol})$. Every new batch was added after at least 6 hours of reaction time, to ensure complete conversion of the previously added TEOS. Together with the second TEOS batch, fluorescein-labeled silica precursor in ethanol $(0.5 \mathrm{~mL}$, $\left.18 \mathrm{mg} \mathrm{mL}{ }^{-1}\left(7.8 \mu \mathrm{mol} \mathrm{mL} \mathrm{mL}^{-1}\right)\right)$ was added. Together with the fourth TEOS batch, rhodamine-labeled silica precursor in ethanol $\left(0.05 \mathrm{~mL}, 20 \mathrm{mg} \mathrm{mL} \mathrm{m}^{-1}\left(10.6 \mu \mathrm{mol} \mathrm{mL}{ }^{-1}\right)\right)$ was added.

The fluorescein-rhodamine-labeled silica microcapsules shown in Fig. 5 were produced with an emulsion consisting of silica microparticles $(1.5 \mathrm{~g}$, diameter $750 \mathrm{~nm})$, water $(12.8 \mathrm{~mL})$ and toluene with $47 \mathrm{mg} \mathrm{mL}{ }^{-1} n$-hexylamine $\left(0.47 \mu \mathrm{mol} \mathrm{mL}^{-1}\right)$ $(150 \mathrm{~mL})$. After the Pickering emulsion was produced 2.5, 3, 2.4, 2.6 and $2 \mathrm{~g}$ batches of TEOS $(12,14,12,13$ and $9.6 \mu \mathrm{mol})$ were added, after at least 6 hours of reaction time, to ensure complete conversion of the previously added TEOS. Together with the second TEOS batch, rhodamine-labeled silica precursor solution in ethanol $\left(0.05 \mathrm{~mL}, 20 \mathrm{mg} \mathrm{mL} \mathrm{m}^{-1}, 10.6 \mu \mathrm{mol} \mathrm{mL}{ }^{-1}\right)$ was added. Together with the fourth TEOS batch, fluorescein-labeled silica precursor solution in ethanol $\left(0.7 \mathrm{~mL}, 18 \mathrm{mg} \mathrm{mL}^{-1}\left(7.8 \mu \mathrm{mol} \mathrm{mL}{ }^{-1}\right)\right)$ was added.

The fluorescein-labeled and rhodamine-labeled silica precursors were synthesized by the addition reaction of (3-aminopropyl)triethoxysilane with rhodamine B isothiocyanate or with fluorescein isothiocyanate, according to van Blaaderen et al. ${ }^{34}$ In short, in $5 \mathrm{~mL}$ anhydrous ethanol, $0.2 \mathrm{mmol}$ of (3-aminopropyl)triethoxysilane (44 mg) was reacted with $0.1 \mathrm{mmol}$ of the dye $(53 \mathrm{mg}$ rhodamine B isothiocyanate or $39 \mathrm{mg}$ fluorescein isothiocyanate). The reaction proceeded for 17 hours in a nitrogen atmosphere at $25^{\circ} \mathrm{C}$.

\section{Imaging studies (light microscopy (LM), scanning electron microscopy (SEM) and fluorescence confocal microscopy (FCM))}

Optical microscopy was used to image the produced Pickering emulsions. The microscopy was performed on a Zeiss Axioplan Universal Microscope. Samples were prepared by placing a droplet of the Pickering emulsion on a glass microscope slide, before placing it on the specimen stage.

Scanning electron microscopy (SEM) was used to examine the silica microcapsules on, amongst other things, morphology and shell thickness. SEM was performed on a FEI Quanta ${ }^{\mathrm{TM}} 3 \mathrm{D}$ FEG low vacuum SEM/focused ion beam (FIB) instrument. The samples were prepared by placing a droplet of the sample on a sample holder covered by double-sided carbon tape. After the 
sample was dried in air, it was sputter-coated with a thin layer of gold, for good conductivity during SEM analysis.

The rhodamine B and fluorescein labelled silica microcapsules were analysed with a fluorescence confocal microscope (FCM). The microscope used for FCM is a Carl Zeiss LSM 780 with Elyra S.1 superresolution platform. The images were made with a $561 \mathrm{~nm}$ (100 mW) laser (red) and with a $488 \mathrm{~nm}$ (100 mW) laser (green).

\section{Interfacial tension}

The interfacial tension between water and toluene, as a function of the $n$-hexylamine concentration in toluene was measured using a Dataphysics DCAT 11 Dynamic Contact Angle Meter and Tensiometer apparatus, a new solution with a predefined concentration was prepared for every measurement. The Wilhelmy plate method was used to determine the surface tension. The Wilhelmy plate was made of Platinum-Iridium and the width of the plate was $19.9 \mathrm{~mm}$. Before use the plate was heated by a flame until it turned red and every measurement was performed at a temperature of $20{ }^{\circ} \mathrm{C}$.

\section{References}

1 A. F. Regin, S. C. Solanki and J. S. Saini, Heat Transfer Characteristics of Thermal Energy Storage System Using PCM Capsules: A Review, Renewable Sustainable Energy Rev., 2008, 12, 2438-2458.

2 R. Vidhyalakshmi, R. Bhakyaraj and R. S. Subhasree, Encapsulation The Future of Probiotics - A Review, Adv. Biol. Res., 2009, 3, 96-103.

3 K. G. H. Desai and H. J. Park, Encapsulation of Vitamin C in Tripolyphosphate Cross-Linked Chitosan Microspheres by Spray Drying, J. Microencapsulation, 2005, 22, 179-192.

4 B. Mishra, B. B. Patel and S. Tiwari, Colloidal Nanocarriers: A Review on Formulation Technology, Types and Applications toward Targeted Drug Delivery, Nanomedicine, 2010, 6, 9-24.

5 M. Li, R. L. Harbron, J. V. M. Weaver, B. P. Binks and S. Mann, Electrostatically Gated Membrane Permeability in Inorganic Protocells, Nat. Chem., 2013, 5, 529-536.

6 M. M. Farid, A. M. Khudhair, S. A. K. Razack and S. Al-Hallaj, A Review on Phase Change Energy Storage: Materials and Applications, Energy Convers. Manage., 2004, 45, 1597-1615.

7 B. Zalba, J. M. Marín, L. F. Cabeza and H. Mehling, Review on Thermal Energy Storage with Phase Change: Materials, Heat Transfer Analysis and Applications, Appl. Therm. Eng., 2003, 23, 251-283.

8 S. Riffat, B. Mempouo and W. Fang, Phase Change Material Developments: A Review, Int. J. Ambient Energy, 2013, 34, 1-14.

9 A. Sharma, V. V. Tyagi, C. R. Chen and D. Buddhi, Review on Thermal Energy Storage with Phase Change Materials and Applications, Renewable Sustainable Energy Rev., 2009, 13, 318-345.

10 W. Krasaekoopt, B. Bhandari and H. Deeth, Evaluation of Encapsulation Techniques of Probiotics for Yoghurt, Int. Dairy J., 2003, 13, 3-13.
11 G. Orive, E. Santos, J. L. Pedraz and R. M. Hernández, Application of Cell Encapsulation for Controlled Delivery of Biological Therapeutics, Adv. Drug Delivery Rev., 2013, 65, 12.

12 C. D. Keating, Inorganic Protocells: Gated Access to Microreactors, Nat. Chem., 2013, 5, 449-451.

13 R. Ciriminna, M. Sciortino, G. Alonzo, A. de Schrijver and M. Pagliaro, From Molecules to Systems: Sol-Gel Microencapsulation in Silica-Based Materials, Chem. Rev., 2011, 111, 765-789.

14 D. Avnir, Organic Chemistry within Ceramic Matrixes: Doped Sol-Gel Materials, Acc. Chem. Res., 1995, 28, 328-334.

15 M. Pagliaro, Silica-Based Materials for Advanced Chemical Applications., RSC Publishing, Cambridge, 2009.

$16 \mathrm{H}$. Mackinney, Microencapsulation by Interfacial Polycondensation, Polym. Eng. Sci., 1963, 485, 71-72.

17 K. Bean, C. F. Black, N. Govan, P. Reynolds and M. R. Sambrook, Preparation of Aqueous Core/silica Shell Microcapsules, J. Colloid Interface Sci., 2012, 366, 16-22.

18 G. Galgali, E. Schlangen and S. van der Zwaag, Synthesis and Characterization of Silica Microcapsules Using a Sustainable Solvent System Template, Mater. Res. Bull., 2011, 46, 2445-2449.

19 Z. Cao, L. Dong, L. Li, Y. Shang, D. Qi, Q. Lv, G. Shan, U. Ziener and K. Landfester, Preparation of Mesoporous Submicrometer Silica Capsules via an Interfacial Sol-Gel Process in Inverse Miniemulsion, Langmuir, 2012, 28, 7023-7032.

20 M. P. Neubauer, M. Poehlmann and A. Fery, Microcapsule Mechanics: From Stability to Function, Adv. Colloid Interface Sci., 2014, 207, 65-80.

21 H. Wang, X. Zhu, L. Tsarkova, A. Pich and M. Möller, All-Silica Colloidosomes with a Particle-Bilayer Shell, ACS Nano, 2011, 5, 3937-3942.

22 J. van Wijk, J. W. O. Salari, N. Zaquen, J. Meuldijk and B. Klumperman, Poly(methyl Methacrylate)-silica Microcapsules Synthesized by Templating Pickering Emulsion Droplets, J. Mater. Chem. B, 2013, 1, 2394-2406.

23 G. Kaptay, On the Equation of the Maximum Capillary Pressure Induced by Solid Particles to Stabilize Emulsions and Foams and on the Emulsion Stability Diagrams, Colloids Surf., A, 2006, 282-283, 387-401.

24 V. M. Masalov, N. S. Sukhinina, E. A. Kudrenko and G. A. Emelchenko, Mechanism of Formation and Nanostructure of Stöber Silica Particles, Nanotechnology, 2011, 22, 275718.

25 J. Schulman and J. Leja, Control of Contact Angles at the Oil-Water-Solid Interfaces. Emulsions Stabilized by Solid Particles (BaSO4), Trans. Faraday Soc., 1954, 598-605.

26 N. Yan, M. R. Gray and J. H. Masliyah, On Water-in-Oil Emulsions Stabilized by Fine Solids, Colloids Surf., A, 2001, 193, 97-107.

27 S. Brandriss and S. Margel, Synthesis and Characterization of Self-Assembled Hydrophobic Monolayer Coatings on Silica Colloids, Langmuir, 1993, 100, 1232-1240.

28 S. A. Kulkarni, S. B. Ogale and K. P. Vijayamohanan, Tuning the Hydrophobic Properties of Silica Particles by Surface 
Silanization Using Mixed Self-Assembled Monolayers, J. Colloid Interface Sci., 2008, 318, 372-379.

29 B. P. Binks, J. A. Rodrigues and W. J. Frith, Synergistic Interaction in Emulsions Stabilized by a Mixture of Silica Nanoparticles and Cationic Surfactant, Langmuir, 2007, 23, 3626-3636.

30 Z. Cui, C. Cui, Y. Zhu and B. Binks, Multiple Phase Inversion of Emulsions Stabilized by in Situ Surface Activation of CaCO3 Nanoparticles via Adsorption of Fatty Acids, Langmuir, 2011, 28, 314-320.

31 W. Stöber, A. Fink and E. Bohn, Controlled Growth of Monodisperse Silica Spheres in the Micron Size Range, J. Colloid Interface Sci., 1968, 69, 62-69.
32 G. Bogush, M. Tracy and C. Z. Iv, Preparation of Monodisperse Silica Particles: Control of Size and Mass Fraction, J. Non-Cryst. Solids, 1988, 104, 95-106.

33 N. Verhaegh and A. Blaaderen, Dispersions of RhodamineLabeled Silica Spheres: Synthesis, Characterization, and Fluorescence Confocal Scanning Laser Microscopy, Langmuir, 1994, 96, 1427-1438.

34 A. van Blaaderen and A. Vrij, Synthesis and Characterization of Colloidal Dispersions of Fluorescent, Monodisperse Silica Spheres, Langmuir, 1992, 81, 2921-2931.

35 J. W. O. Salari, G. T. Jemwa, H. M. Wyss and B. Klumperman, Reconstruction of the 3D Structure of Colloidosomes from a Single SEM Image, Soft Matter, 2011, 7, 2033-2041. 